\title{
Visualization of PAX7 protein dynamics in muscle satellite cells in a YFP knock-in- mouse line
}

\author{
Yasuo Kitajima ${ }^{1,2}$ and Yusuke Ono ${ }^{1,3^{*}}$ (D)
}

\begin{abstract}
Background: Satellite cells are residential muscle stem cells that express a paired box protein, PAX7.

Results: Here, we report a knock-in mouse line expressing a PAX7-enhanced yellow fluorescent protein (YFP) fusion protein that enables visualization of PAX7 protein dynamics in living satellite cells through YFP fluorescence. The YFP fluorescence signals in Pax7-YFP knock-in mice clearly recapitulated the endogenous expression of PAX7 protein in satellite cells. YFP+ satellite cells were efficiently isolated from muscle tissues by fluorescence-activated cell sorting. Homozygous Pax7-YFP knock-in mice (Pax $7^{\text {YFP } \text { YFP }}$ ) were viable, grew and regenerated muscle normally, and Pax $7^{\text {YFP YFP }}$ mouse-derived satellite cells proliferated, differentiated, and self-renewed as efficiently as those from wild-type $\left(\operatorname{Pax} 7^{+/+}\right)$mice.

Conclusions: Taken together, our Pax7-YFP mouse line is a useful tool to aid the development of stem-cell-based therapies for muscle diseases.
\end{abstract}

Keywords: Pax7, Knock-in mouse, YFP, Myogenesis, Muscle regeneration

\section{Background}

Skeletal muscle retains a remarkable capacity to regenerate. This regenerative capacity depends on residential stem cells called muscle satellite cells that provide myonuclei not only for regeneration in the adult but also for postnatal muscle growth [1-6]. Satellite cells are located between the basal lamina and the plasmalemma of myofibers and are mitotically quiescent in healthy adult muscle [7]. Transplantation analysis shows that satellite cells possess potent myogenic and self-renewal abilities to reconstitute host muscle in vivo [8-13]. In contrast, depletion of the satellite cell population by inducing expression of diphtheria toxin fragment A [14-16] or a failure of satellite cell function [17] results in severe loss of regenerated muscle. Together, these data provide

\footnotetext{
* Correspondence: yusuke-ono@nagasaki-u.ac.jp

${ }^{1}$ Musculoskeletal Molecular Biology Research Group, Basic and Translational Research Center for Hard Tissue Disease, Nagasaki University Graduate School of Biomedical Sciences, 1-7-1 Sakamoto, Nagasaki 852-8588, Japan ${ }^{3}$ Agency for Medical Research and Development (AMED), 1-7-1 Otemachi, Chiyodaku, Tokyo 100-0004, Japan

Full list of author information is available at the end of the article
}

direct evidence that the satellite cell population is indispensable for regeneration in adult muscle.

Satellite cells are normally quiescent but become activated in response to stimulation including traumatic muscle injury. After activation, they enter the cell cycle and give rise to proliferative satellite-cell progeny, called myoblasts. Myoblasts then undergo myogenic differentiation and either fuse with existing myofibers or form new myofibers by producing myonuclei. Meanwhile, a minority of the population return to a quiescent state to self-renew, maintaining the stem-cell pool $[8,12,18-21]$.

The paired box protein, PAX7, is a transcription factor that is uniformly expressed in quiescent to proliferative state satellite cells. However, it is downregulated during myogenic differentiation [18, 20]. PAX7 plays important roles in satellite cell survival, specification, proliferation, and differentiation [19, 22-28]. Mice lacking the $\operatorname{Pax} 7$ gene are viable until 2-3 weeks after birth with a marked reduction in body-size [23, 27]. Pax7-null mice exhibit a progressive loss of satellite cells in muscle during growth, because of a decrease in proliferation and precocious myogenesis, leading to a significant decrease in myonuclear-numbers and myofiber-diameters [23, 25, 27, 29]. More recently, 
satellite-cell-specific inactivation of Pax7 induced by tamoxifen injection in mice resulted in a reduced satellite cell number, a proliferative defect, and precocious myogenic differentiation, resulting in a severe impairment in muscle regeneration [30-32]. Together, these findings illustrate that PAX7 expressed in satellite cells is essential not only during the juvenile period to give rise to progeny but also during muscle regeneration in adults [30, 31, 33].

Here, we generated a mouse line carrying the PAX7 protein fused with enhanced yellow fluorescent protein (YFP) that enables indirect visualization of endogenous PAX7 protein dynamics in living satellite cells. YFP+ satellite cells could be efficiently isolated by fluorescence-activated cell sorting (FACS) without antibody staining and were transplantable, similarly to cells isolated from transgenic Pax7-ZsGreen, Pax7-nGFP, and Pax7-GFP reporter mice that have recently been reported [34-36]. Importantly, the YFP-tag does not interfere with the function of the endogenous PAX7 protein because Pax ${ }^{\text {YFP/YFP }}$ homozygous mice are born, grow, and regenerate muscle normally, and Pax $7^{\text {YFP/YFP }}$ mouse-derived satellite cells undergo proliferation, myogenic differentiation, and self-renewal, similar to wild-type satellite cells. Although the fluorescence intensity of YFP-tagged PAX7 protein is lower than other reporter lines, our Pax7-YFP mouse line allows not only further characterization of satellite cell dynamics but also the visualization and biochemical analysis of endogenous PAX7 protein dynamics. Thus, our newly established knock-in mouse line will be an additional useful tool for the researchers in the field of muscle biology and facilitate the development of stem-cell-based therapies for muscle diseases.

\section{Methods}

\section{Antibodies and reagents}

Antibodies and reagents were obtained from the following sources. PE-conjugated anti-CD31, anti-CD45, and anti-Sca-1 and APC-conjugated anti-Vcam1 antibodies were obtained from BioLegend (San Diego, CA, USA). Rabbit or mouse anti-GFP antibodies cross-reacting with YFP were obtained from Thermo Fisher Scientific (Carlsbad, CA, USA) or EMD Millipore. Mouse anti-PAX7 and mouse anti-myosin heavy chain (MF20, MAB4470) antibodies were purchased from R\&D Systems (Minneapolis, MN, USA). Rabbit anti-MyoD antibody was from Santa Cruz Biotechnology (Santa Cruz, CA, USA). Rabbit anti-Laminin antibody was obtained from Sigma (Sigma-Aldrich, St. Louis, MO). Rat anti-Laminin $\alpha 2$ antibody was obtained from Enzo (Enzo Life Sciences, NY). Rabbit anti-Dystrophin antibody was obtained from Abcam (Cambridge, MA, USA). Rat anti-Ki67 antibody and DAKO Protein Block were obtained from DAKO (Tokyo, Japan). Alexa Fluor-conjugated secondary antibodies were purchased from Thermo Fisher Scientific. M.O.M. kit and mounting medium containing 4,6-diamidino-2-phenylindole (DAPI) for nuclear staining was obtained from Vector Laboratories (Burlingame, CA, USA).

\section{Generation of Pax7-YFP knock-in mouse line}

The Experimental Animal Care and Use Committee of Nagasaki University approved all animal experimentation used in this study (ref. no. 1203190970). The BRUCE-4 ES cell line (C57/BL6J) was used to generate the Pax7-YFP knock-in mouse line. A targeting vector was generated to modify the $\operatorname{Pax} 7$ gene by inserting an EYFP sequence downstream of the terminal exon 9 of $\operatorname{Pax} 7$ (Fig. 1a). To express a Pax7-YFP fusion protein, the only stop codon of exon 9 was deleted. Briefly, an EYFP-loxP flanked Neo cassette was replaced with the terminal exon 9 of $\operatorname{Pax} 7$ to construct the Pax7-YFP knock-in vector. The Neo cassette was not removed. The genotype of the transgenic Pax7-YFP knock-in (KI) mice was verified by PCR using the following primer pair (Fig. 1b); forward primer 5'-AGCGCCGTA TGAAGCTTGGG-3', reverse primer 5'-AAGGGGACT GAGGTGAGGAGA-3', (wild-type = 134 bp, Pax7-YFP = $2441 \mathrm{bp}$ ). Male mice between 7 and 14 weeks of age were used in all experiments.

\section{Muscle injury}

Cardiotoxin (CTX, Sigma-Aldrich) was prepared by dissolving a freshly opened tube in $0.9 \% \mathrm{NaCl}$ at $10 \mu \mathrm{M}$. To induce muscle injury, $100 \mu \mathrm{l}$ of $10 \mu \mathrm{M}$ CTX was injected intramuscularly into the tibialis anterior (TA) muscle of anesthetized mice. Regenerating muscles were isolated at 3, 7, and 14 days after CTX injection. TA muscles were frozen in either 2-methylbutane cooled with liquid nitrogen or liquid nitrogen for histological analysis or RNA isolation, respectively, and stored at $-80{ }^{\circ} \mathrm{C}$. Transverse muscle sections were cut using a cryostat.

\section{Transplantation}

Dystrophin-null mdx mutant mice, a mouse model for Duchenne muscular dystrophy (DMD), were used as a recipient animal. Regeneration of TA muscle in mdx mice was induced by intramuscular injection of $100 \mu \mathrm{l}$ CTX 1 day before transplantation. Pax $7^{\mathrm{YFP} / \mathrm{YFP}}$ mice were used as a donor animal. $\mathrm{YFP}^{+}$satellite cells $\left(5 \times 10^{4}\right.$ cells $)$ were freshly isolated from $\operatorname{Pax} 7^{\mathrm{YFP} / \mathrm{YFP}}$ mice by FACS and were transplanted into CTX-pretreated TA muscles of $\mathrm{mdx}$ mice. TA muscles were harvested 2 weeks after transplantation and stored at $-80^{\circ} \mathrm{C}$.

\section{FACS analysis}

Hindlimb muscles were collected and excess fat, connective tissue, and tendons removed. Mononuclear cells 


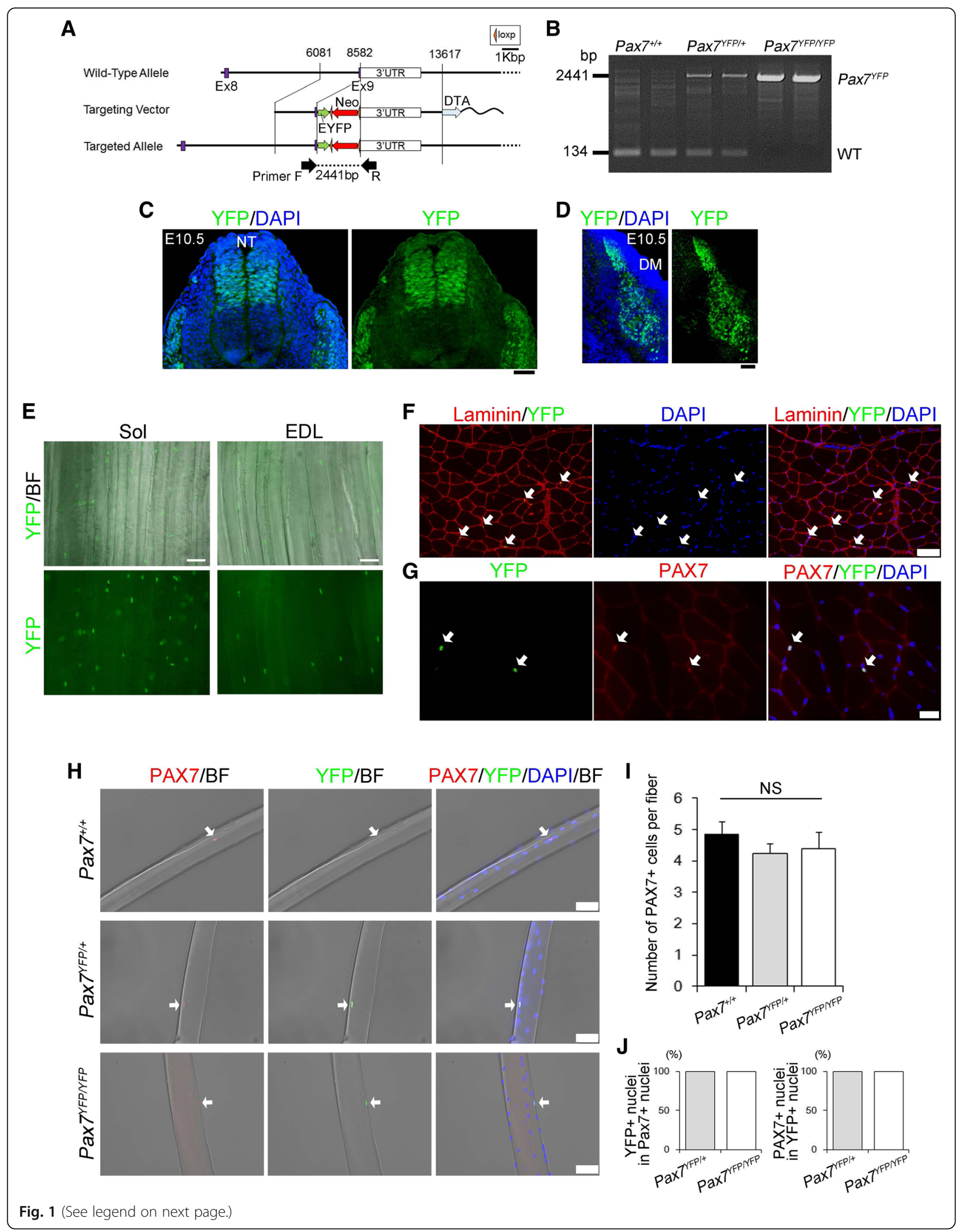




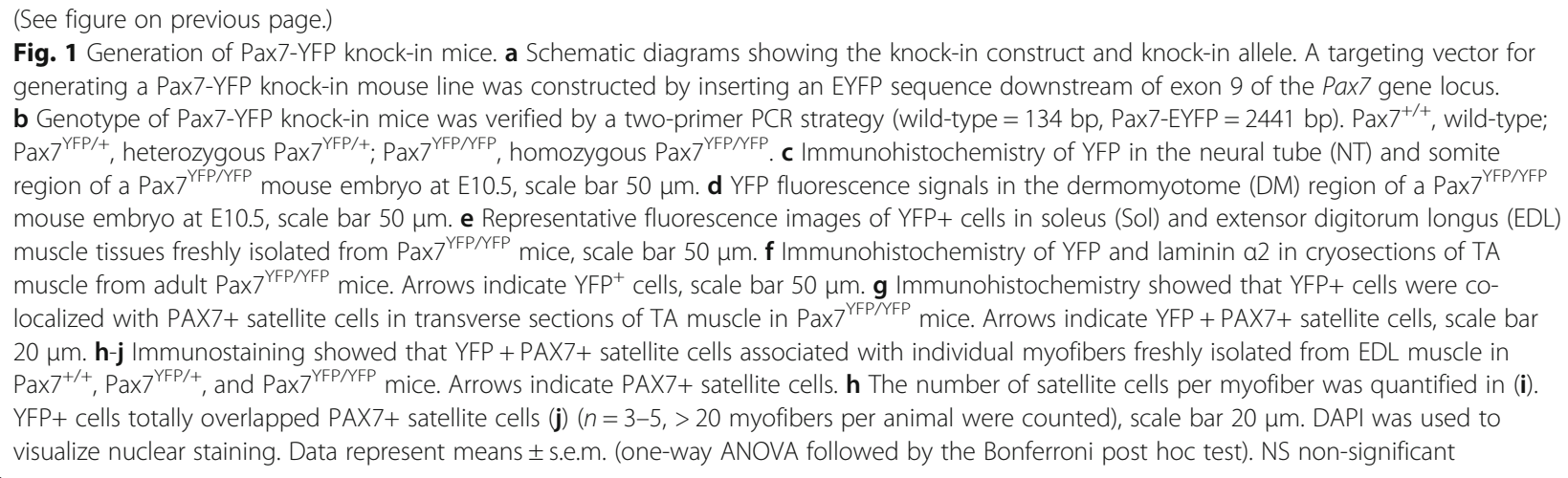

from hindlimb muscles were prepared using $0.2 \%$ collagenase type II (Worthington Biochemical) as previously described [37]. Mononuclear cells were stained with PE-conjugated anti-CD31, anti-CD45, and anti-Sca-1 and APC-conjugated anti-Vcam1 antibodies on ice for 30 min and resuspended in PBS containing 2\% FBS. Cell sorting was performed using a FACS Aria II flow cytometer (BD Immunocytometry Systems). Debris and dead cells were excluded by forward scatter, side scatter, and PI gating. Data were collected using FACS Diva software (BD Biosciences).

\section{Myofiber and satellite cell isolation and culture}

Individual myofibers were isolated from the extensor digitorum longus (EDL) muscles, as described previously [38]. In brief, EDL muscles were digested using $0.2 \%$ type I collagenase (Worthington Biochemical) in DMEM for 90 min at $37{ }^{\circ} \mathrm{C}$ under $5 \% \mathrm{CO}_{2}$. For immunocytochemical analysis, EDL myofibers were immediately fixed using $4 \%$ paraformaldehyde (PFA). Satellite cells were obtained from isolated myofibers and cultured in growth medium (GM; DMEM supplemented with $30 \%$ fetal bovine serum, $1 \%$ chicken-embryo extract, $10 \mathrm{ng} / \mathrm{ml}$ basic fibroblast growth factor, and $1 \%$ penicillin-streptomycin) at $37{ }^{\circ} \mathrm{C}$ under $5 \% \mathrm{CO}_{2}$ immediately after isolation. Myogenic differentiation was induced in differentiation medium (DM; DMEM supplemented with $5 \%$ horse serum and $1 \%$ penicillin-streptomycin) at $37^{\circ} \mathrm{C}$ under $5 \% \mathrm{CO}_{2}$.

\section{Immunostaining}

Immunocytochemistry of satellite cells and isolated single myofibers was performed as described previously [38]. Samples were fixed with $4 \%$ PFA, blocked/permeabilized with phosphate-buffered saline containing $0.3 \%$ Triton X-100 and 5\% goat serum for $20 \mathrm{~min}$ at room temperature, and incubated with primary antibodies at $4{ }^{\circ} \mathrm{C}$ overnight. For immunohistochemistry, cryosections of TA muscle tissues and E10.5 embryos were fixed with $4 \%$ PFA, blocked with 5\% goat serum or the M.O.M kit (Vector Laboratories) for $30 \mathrm{~min}$ at room temperature and incubated with primary antibodies at $4{ }^{\circ} \mathrm{C}$ overnight. All immunostained samples were visualized using appropriate species-specific Alexa Fluor fluorescenceconjugated secondary antibodies. Samples were viewed on an Olympus IX83 microscope (Olympus, Tokyo, Japan) or on a Cell Insight CX5 (Thermo Fisher Scientific). Digital images were acquired and quantified with a DP80 camera using cellSens software (Olympus) or with a Photometrics X1 camera using HCS Studio software (Thermo Fisher Scientific). Images were optimized globally and assembled into figures using Adobe Photoshop. Immunostaining for laminin to measure cross-sectional area (CSA) of centrally nucleated regenerating myofibers was performed, and CSA was quantified using cellSens software (Olympus). For EdU detection, the Click chemical reaction was performed after primary and secondary staining according to the manufacturer's instructions using a Click-iT EdU Imaging Kit (Thermo Fisher Scientific).

\section{Quantitative revere transcription-PCR (Q-PCR)}

Total RNA was isolated using an RNAeasy Kit (Qiagen, Hilden, Germany). For real-time PCR, first-strand cDNA was synthesized using oligo-dT primers (Toyobo). The expression levels of selected genes were analyzed using a CFX96 real-time PCR detection system (Bio-Rad, Tokyo, Japan) according to the manufacturer's instructions. Primer sequences were listed in Additional file 1.

\section{Statistical analysis}

Statistical analyses were performed with SPSS software (IBM) to determine significant differences from a two-tailed distribution using the paired or unpaired Student's $t$ test. In comparisons of more than two groups, non-repeated measures analysis of variance (ANOVA) followed by the Bonferroni post hoc test was used. $P$ values are indicated on each figure as $<0.05(*)$, $<0.01{ }^{(* *)}$ ), and $<0.0011^{(* * *)}$. All error bars are indicated as means \pm s.d. or s.e.m. NS indicates statistically non-significant. 


\section{Results}

\section{Generation of a PAX7-YFP mouse line}

To generate a mouse line expressing a PAX7 and YFP fusion protein (PAX7-YFP), we constructed a targeting vector with the enhanced YFP gene inserted into the end of exon 9 of the endogenous Pax7 locus with the endogenous Pax7 stop codon deleted (Fig. 1a, b). The Pax7 gene is highly expressed in the craniofacial region and somites [34, 39, 40]. Consistent with the expression pattern of PAX7 in mouse embryos [22, 24], immunohistochemistry showed that YFP-positive cells were localized in the neural tube and dermomyotome in homozygous (Pax7 ${ }^{\text {YFP/YFP }}$ ) E10.5 embryos (Fig. 1c, d). Although native YFP fluorescence signal recapitulated the endogenous expression pattern of PAX7 in whole homozygous embryos, its fluorescence intensity was not strong enough to detect the defined PAX7-expressing areas in the somites under the fluorescence stereo microscopy (data not shown).

To determine whether the YFP fluorescence signal recapitulated the localization of endogenous PAX7 protein in adult muscle, freshly isolated soleus (Sol), extensor digitorum longus (EDL), and tibialis anterior (TA) muscles were obtained from 12-week-old Pax $7^{\text {YFP/YFP }}$ mice immediately after sacrifice. YFP-positive signals were detected between individual myofibers, which is the expected localization of satellite cells (Fig. 1e). In general, the Sol muscle contains more satellite cells than other limb muscles such as TA and EDL muscles [8, 10], indicating that the YFP-positive signals accurately represent the number of satellite cells per myofiber in mouse muscles. Immunohistochemical analysis showed co-localization between $\mathrm{YFP}^{+}$ nuclei and $\mathrm{PAX7}^{+}$nuclei in cross-sections of TA muscle (Fig. 1f, g). Importantly, all $\mathrm{YFP}^{+}$nuclei corresponded to $\mathrm{PAX}^{+}$nuclei of satellite cells located between myofibers of the EDL muscle in both $\operatorname{Pax} 7^{\mathrm{YFP} /+}$ and $\operatorname{Pax} 7^{\mathrm{YFP} / \mathrm{YFP}}$ mice (Fig. 1h-j). These data indicate that expression of the PAX7-YFP fusion protein faithfully recapitulated the expression pattern of PAX7 protein, allowing us to indirectly and accurately detect the dynamics of endogenous PAX7 expression via YFP detection.

\section{Isolation of the satellite cell population from Pax7-YFP knock-in mice by FACS}

We next validated whether YFP+ satellite cells in Pax $7^{\mathrm{YFP} /+}$ mice can be purified by FACS. For satellite-cell-sorting, a $\mathrm{CD} 1^{-} \mathrm{CD} 45^{-} \mathrm{Sca1}^{-} \mathrm{Vcam}^{+}$mononuclear fraction [41-43] was obtained from limb muscles of wild-type mice (Fig. 2a-c). FACS analysis showed that $98.8 \%$ of YFP+ cells sorted from Pax $7^{\mathrm{YFP} /+}$ mice by FACS overlapped with the $\mathrm{CD} 31^{-} \mathrm{CD} 45^{-} \mathrm{Sca} 1^{-} \mathrm{Vcam}^{+}$mononuclear fraction (Fig. 2d, e), indicating that the satellite cell fraction could be isolated from $\operatorname{Pax} 7^{\mathrm{YFP} /+}$ mice with YFP fluorescence without antibody staining.
Immunostaining also confirmed that a FACS-sorted YFP+ cell fraction contained $97.2 \%$ PAX7+ or $98.4 \%$ MyoD+ cells (Fig. 2f-j). Thus, our validation demonstrated that the satellite cell population could be prospectively and efficiently isolated from muscles of $\operatorname{Pax} 7^{\mathrm{YFP} /+}$ mice by FACS.

\section{YFP mirrors the dynamics of endogenous PAX7 in satellite cells}

In normal muscle regeneration, satellite cells are activated, proliferated, and then committed to myogenic differentiation to give rise to newly formed myofibers. To examine whether expression dynamics of the Pax7-YFP fusion gene mirrors endogenous Pax 7 gene expression during muscle regeneration, muscle injury was induced by intramuscular injection of CTX into the TA muscle. We compared the expression levels of the endogenous $\operatorname{Pax} 7$ gene in regenerating muscles of wild-type mice with those of the $Y F P$ gene from $\operatorname{Pax} 7^{\mathrm{YFP} /+}$ mice. Expression patterns of YFP and Pax7 genes were highly similar between $P a x 7^{+/+}$and $\operatorname{Pax} 7^{\mathrm{YFP} /+}$ mice with peak expression at day 3 following CTX injection (Fig. 3a-f), when activated satellite cell progeny normally undergo population expansion [44]. Furthermore, we confirmed that $\mathrm{YFP}^{+}$satellite cells in regenerative muscles were clearly detectable by immunohistochemistry (Fig. 3g, h).

Next, we examined whether the coincident expression of YFP and Pax7 was also observed in plated satellite cells during myogenic progression. Satellite cells were isolated from limb muscles of Pax $7^{\mathrm{YFP} /+}$ mice by FACS and maintained in growth medium (GM) before induction of myogenic differentiation using differentiation medium (DM) for 3 days (Fig. 3i). Q-PCR analysis demonstrated a strong correlation between Pax7 and YFP mRNA levels during myogenic progression (Fig. 3j-o). Moreover, co-immunostaining for PAX7 and YFP revealed almost identical fluorescence levels in plated satellite cells under GM conditions (Fig. 3p). Taken together, our data illustrate mirrored dynamics between PAX7 and YFP in satellite cells isolated from $\operatorname{Pax} 7^{\mathrm{YFP} /+}$ mice.

\section{Pax7-YFP homozygous mice grow and regenerate muscle normally}

Targeted disruption of $\operatorname{Pax} 7$ in mice leads to a postnatal growth defect and death at 2-3 weeks after birth [23, 25, 27, 29]. In Pax7-deficient mice, the number of satellite cells is progressively lost in postnatal stages, and thus, fiber-diameters are significantly reduced [23, 25, 27, 29]. Satellite-cell-specific inactivation of Pax7 in mice results in a loss of satellite cells, reduced proliferation ability, and precocious myogenic differentiation, thus leading to severe impairment of muscle regeneration [30-32]. In the present study, we examined whether the function of $\operatorname{Pax} 7$ was 


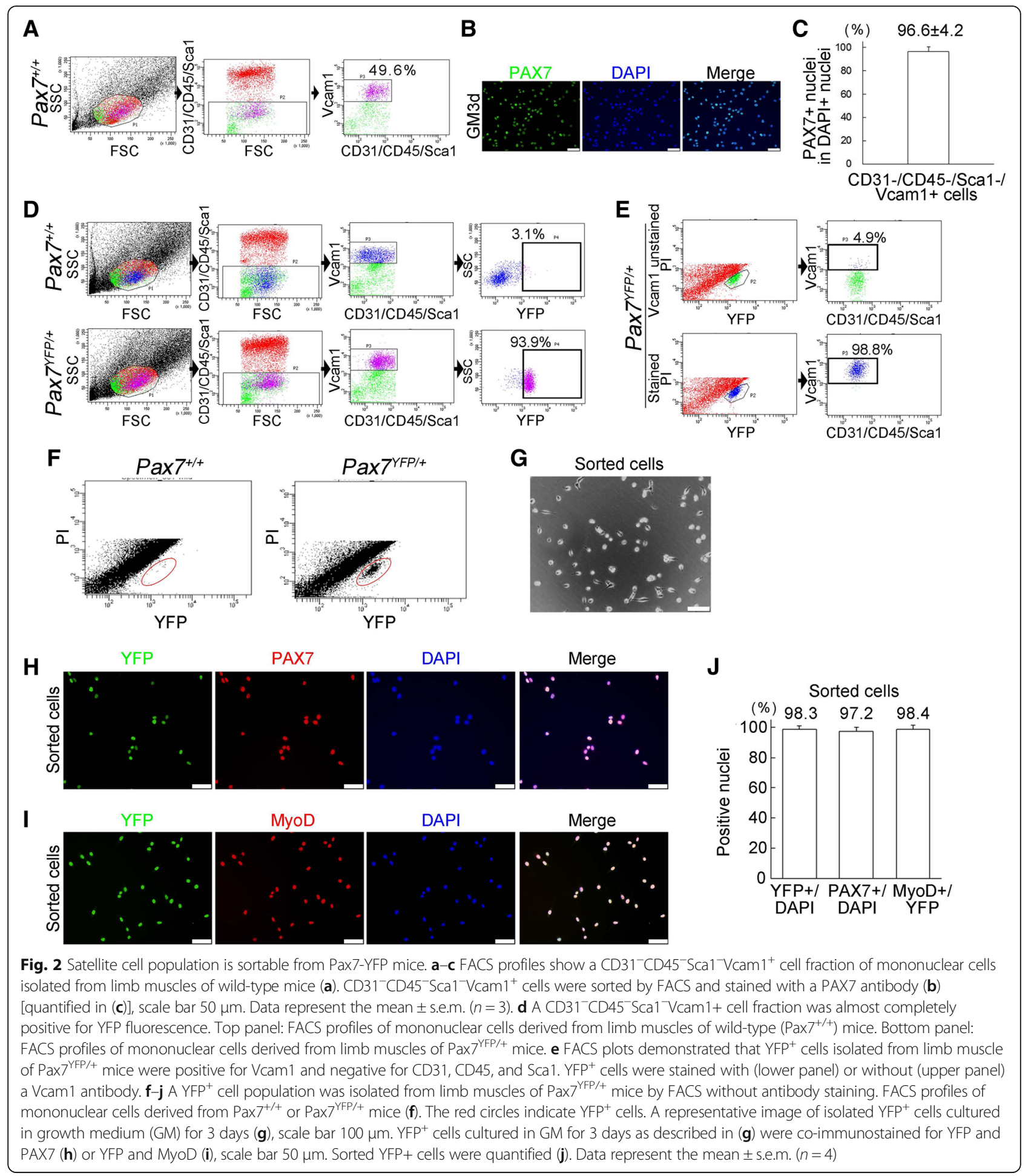

maintained in satellite cells in Pax7-YFP homozygous $\left(\operatorname{Pax} 7^{\mathrm{YFP} / \mathrm{YFP}}\right)$ mice. Muscle regeneration was induced by CTX injection into the TA muscle of Pax7-YFP knock-in mice, and regenerating muscles were removed 2 weeks after CTX injection. Immunohistochemical analysis showed that the cross-sectional area
(CSA) and muscle weight of regenerated muscles from $\operatorname{Pax} 7^{\text {YFP/YFP }}$ mice were both similar to those of $\mathrm{Pax}^{+/+}$or $\mathrm{Pax} 7^{\mathrm{YFP} /+}$ mice (Fig. $4 \mathrm{a}-\mathrm{c}$ ).

Having shown that muscle regeneration was unlikely to be disturbed by expression of the Pax7-YFP fusion protein, we further determined whether Pax7-YFP KI 


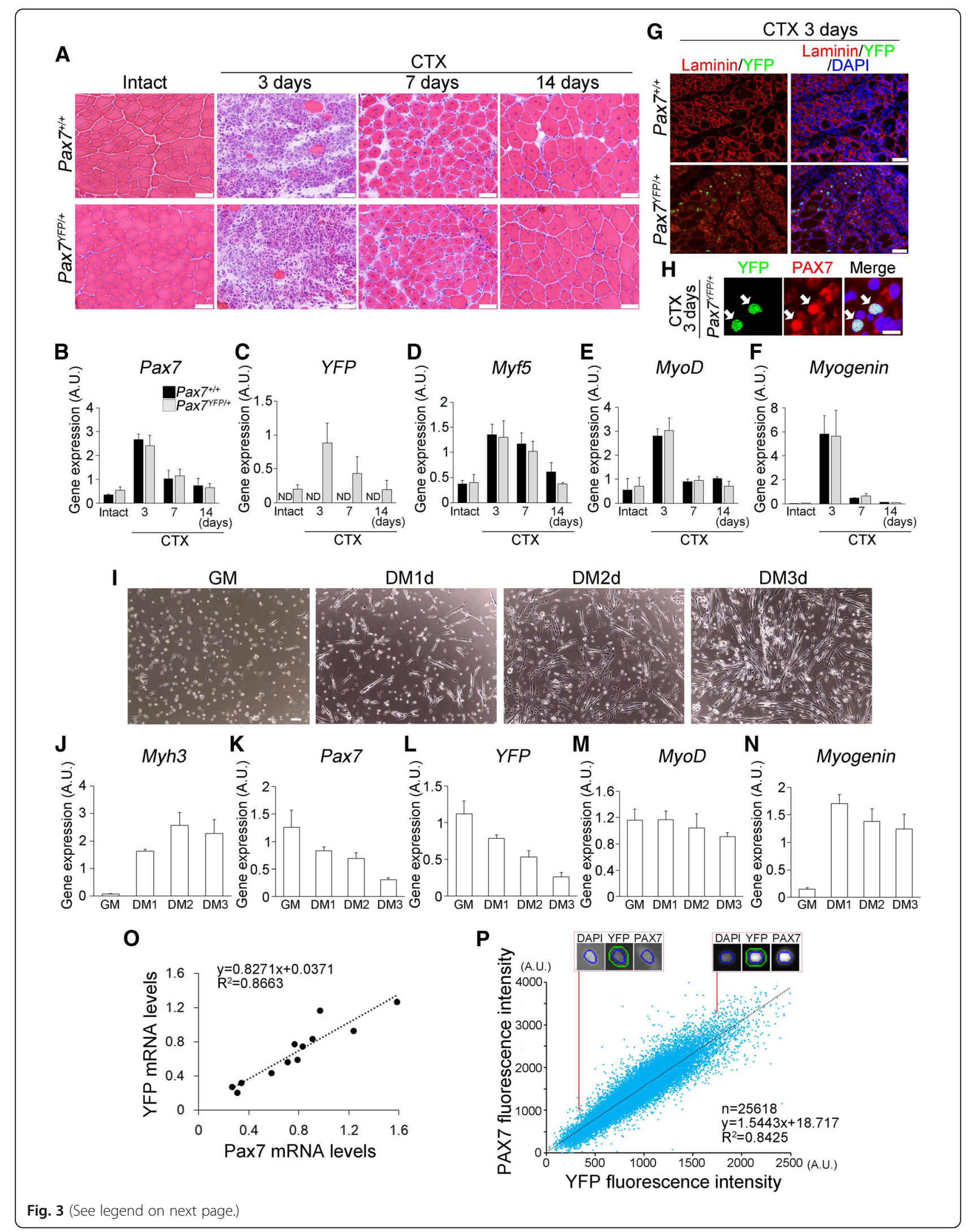


(See figure on previous page.)

Fig. 3 YFP expression levels mirror the endogenous dynamics of PAX7. $\mathbf{a}-\mathbf{h}$ Regeneration of the TA muscle was induced by injection of cardiotoxin (CTX). H\&E staining of cross-sections of TA muscle in Pax $7^{+/+}$and Pax $7^{\mathrm{YFP} /+}$ mice, $0,3,7$, and 14 days following CTX injection, scale bar 50 um (a). Q-PCR analysis of gene expression dynamics of myogenic regulatory factors (Pax7, Myf5, MyoD, and Myogenin) and YFP during muscle regeneration $(\mathbf{b}-\mathbf{f})$. Data represent means \pm s.e.m. (ND, not detected; $n=3-5$, per group). $\mathbf{g}$ Immunohistochemistry for YFP and Laminin a2 proteins in cross-sections of TA muscle isolated from Pax $7^{+/+}$or Pax ${ }^{Y F P /+}$ mice at day 3 after CTX injection, scale bar $50 \mu m$. $\mathbf{h}$ Immunohistochemistry for YFP and PAX7 proteins in cross-sections of TA muscle isolated from Pax7 $7^{\text {YFP/+ }}$ mice at day 3 after CTX injection, scale bar $10 \mu \mathrm{m}$. $\mathbf{i}-\mathbf{o}$ Satellite cells were isolated from limb muscles of Pax $7^{\mathrm{YFP} /+}$ mice and cultured in growth medium (GM). Myogenic differentiation was induced by differentiation medium (DM) for 3 days. Representative images are shown (i), scale bar $100 \mu \mathrm{m}$. $\mathbf{j}$-o Q-PCR analysis shows a positive correlation between Pax7 and YFP mRNA levels during myogenic differentiation [quantified in $(\mathbf{o})\left(R^{2}=0.8663 ; n=12\right)$ ]. Data represent the mean \pm s.d. ( $n=3$, each group). $\mathbf{p}$ Co-immunostaining revealed a positive correlation between YFP and PAX7 protein levels in satellite cells maintained under GM conditions. Fluorescence intensity was measured using the Celllnsight CX5 Platform

mouse-derived satellite cells undergo normal myogenic progression in culture ex vivo. The YFP+ satellite cell population was isolated from limb muscles of $\operatorname{Pax} 7^{\mathrm{YFP} / \mathrm{YFP}}$ mice and then cultured under GM conditions. Myogenic differentiation was then induced by changing the medium to DM for culturing for 3 days (Fig. 4d). To evaluate proliferation, the EdU pulse-chase assay was performed under GM conditions. The proportion of EdU+ satellite cells from $\operatorname{Pax} 7^{\text {YFP/YFP }}$ mice was not different from that of $\mathrm{Pax}^{+/+}$mice (Fig. 4e, f). We also confirmed that $\operatorname{Pax} 7^{\text {YFP/YFP }}$ mouse-derived satellite cells undergo myogenic differentiation (Fig. 4g, h) and self-renewal (Fig. 4i, j), similar to $\mathrm{Pax}^{+/+}$cells. Therefore, our results indicate that the Pax7-YFP fusion protein does not interfere with satellite cell functions, and thus, $\operatorname{Pax} 7^{\mathrm{YFP} / \mathrm{YFP}}$ mice efficiently regenerate muscle after injury as well as wild-type mice.

In mdx mice, transplanted satellite cells give rise to progeny in the regenerating niche and reconstitute myofibers [8-13]. We sought to determine whether Pax7-YFP knock-in mouse-derived satellite cells could also be transplanted into limb muscle and regenerate myofibers and self-renew in the host muscle. Satellite cells were isolated from the limb muscles of Pax $7^{\text {YFP/YFP }}$ mice by FACS, and $5 \times 10^{4}$ YFP+ cells were grafted into regenerating TA muscle of mdx mice, which had been injected with CTX 1 day prior to the transplantation (Fig. 4k). Non-transplanted muscles were used as a control. Muscles were removed 14 days following transplantation, and transverse sections were immunostained. YFP+ donor-derived satellite cells were detected in the satellite cell niche surrounded by basal lamina (Fig. 4l), and their contribution to muscle regeneration was visualized by dystrophin-expressing myofibers accompanied by YFP+ donor-derived satellite cells (Fig. $4 \mathrm{~m}$ ). These data indicated that Pax7-YFP knock-in mouse-derived satellite cells are transplantable and give rise to newly formed myofibers in regenerating muscles.

\section{Discussion}

The transcription factor PAX7 is an established marker for satellite cells in adult skeletal muscle. Here, we generated a novel knock-in mouse line to enable visualization of PAX7 protein via YFP fluorescence in living satellite cells. Our comprehensive analysis of Pax7-YFP mice demonstrated that YFP fluorescence levels accurately recapitulate endogenous PAX7 protein levels and that all quiescent and undifferentiated satellite cells express YFP protein. YFP+ satellite cells are clearly detectable by immunohistochemistry of cross-sections of both intact and injured muscle tissues in Pax7-YFP knock-in mice, even in the inflammatory stages during muscle regeneration. Also of importance, Pax7 $7^{\text {YFP/YFP }}$ homozygous mice are born, grow, and regenerate muscle normally. Satellite cells isolated from Pax7 $7^{\text {YFP/YFP }}$ mice proliferate, differentiate, and self-renew as well as those from wild-type Pax $7^{+/+}$mice, indicating that the YFP-tag does not interfere with the function of the endogenous PAX7 protein.

The satellite cell population can be isolated from skeletal muscle tissue of adult mice using satellite-cell-specific cell surface markers (e.g., Vcam1 and $\alpha 7$-integrin) combined with non-myogenic markers (e.g., CD45 and CD31) $[11,12,41-43]$. In the present study, we demonstrated that satellite cells can be highly purified from muscle tissues of Pax7-YFP knock-in mice by FACS without antibody staining, similar to cells isolated from transgenic Pax7-ZsGreen, Pax7-nGFP, and Pax7-GFP reporter mouse lines that have recently been reported [34-36]. Recently, Rocheteau et al. reported that a $\operatorname{Pax} 7^{\text {high }}$ cell population retains stemness in quiescent satellite cells using Pax7-nGFP reporter adult mice [35]. Our Pax7-YFP knock-in mouse line could be applicable to further characterize the stem-like population. FACS isolated YFP + satellite cells from our mouse line are also transplantable into regenerating muscle of $\mathrm{mdx}$ mice and give rise to progeny as well as newly formed myofibers. Indeed, we believe Pax7-YFP mice will be useful for developing stem-cell-based therapies for muscle diseases.

\section{Conclusions}

We established a Pax7-YFP knock-in mouse line to further understand the function and dynamics of PAX7 protein in satellite cells. This knock-in mouse line is 

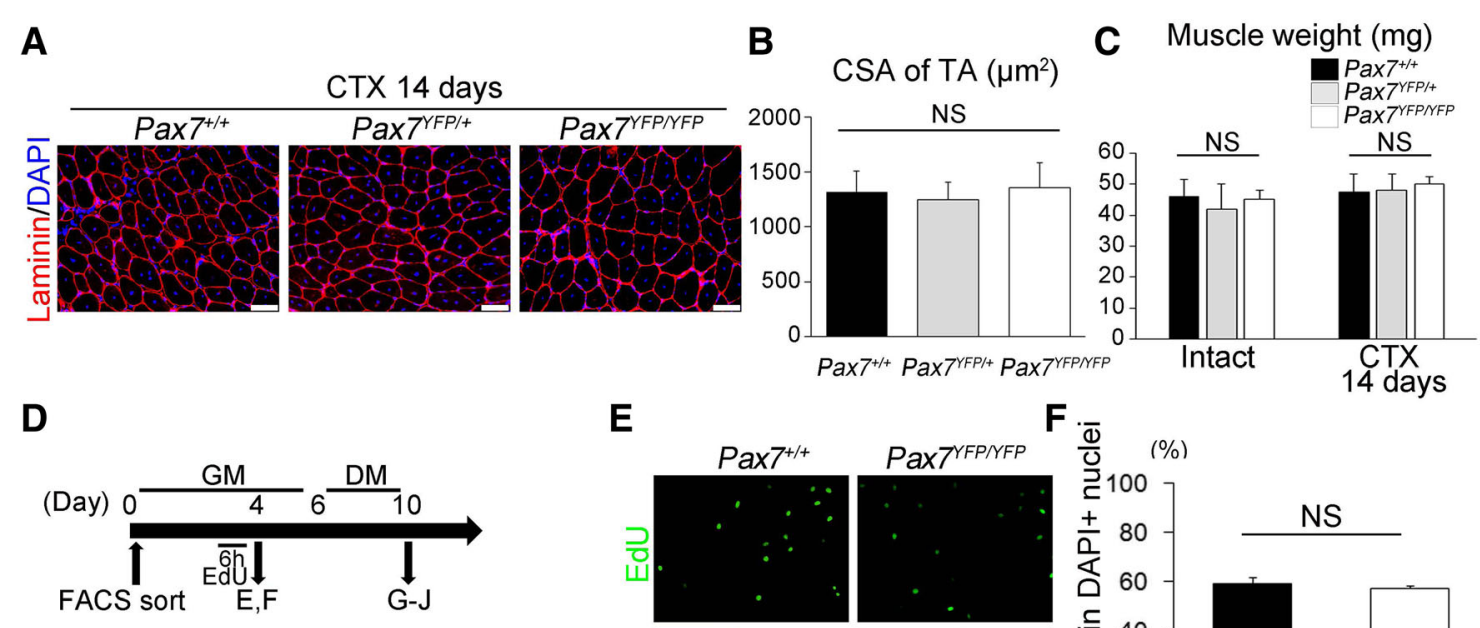

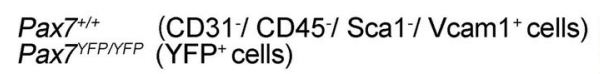
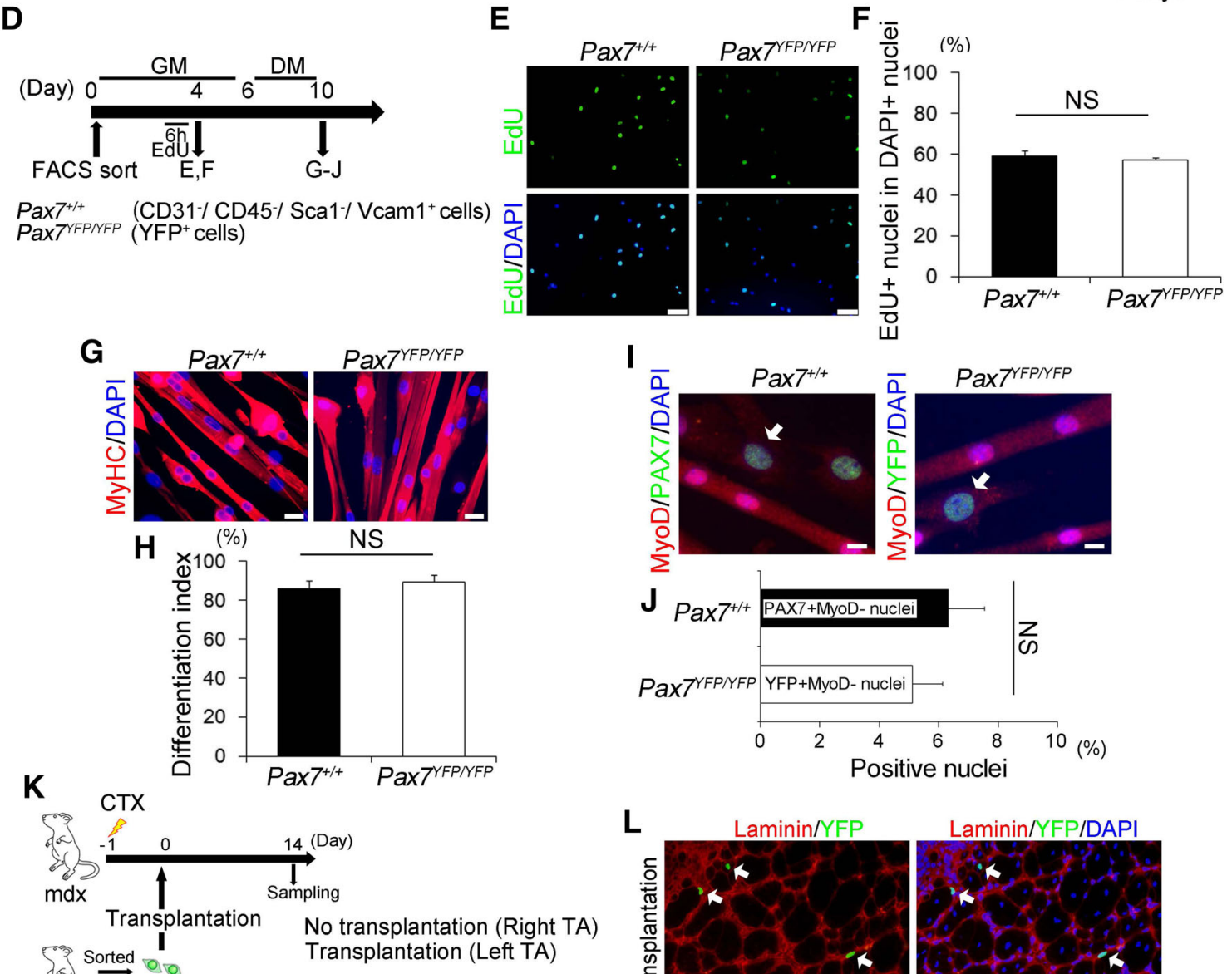

A $\longrightarrow$ o

PaX TYFPYYP
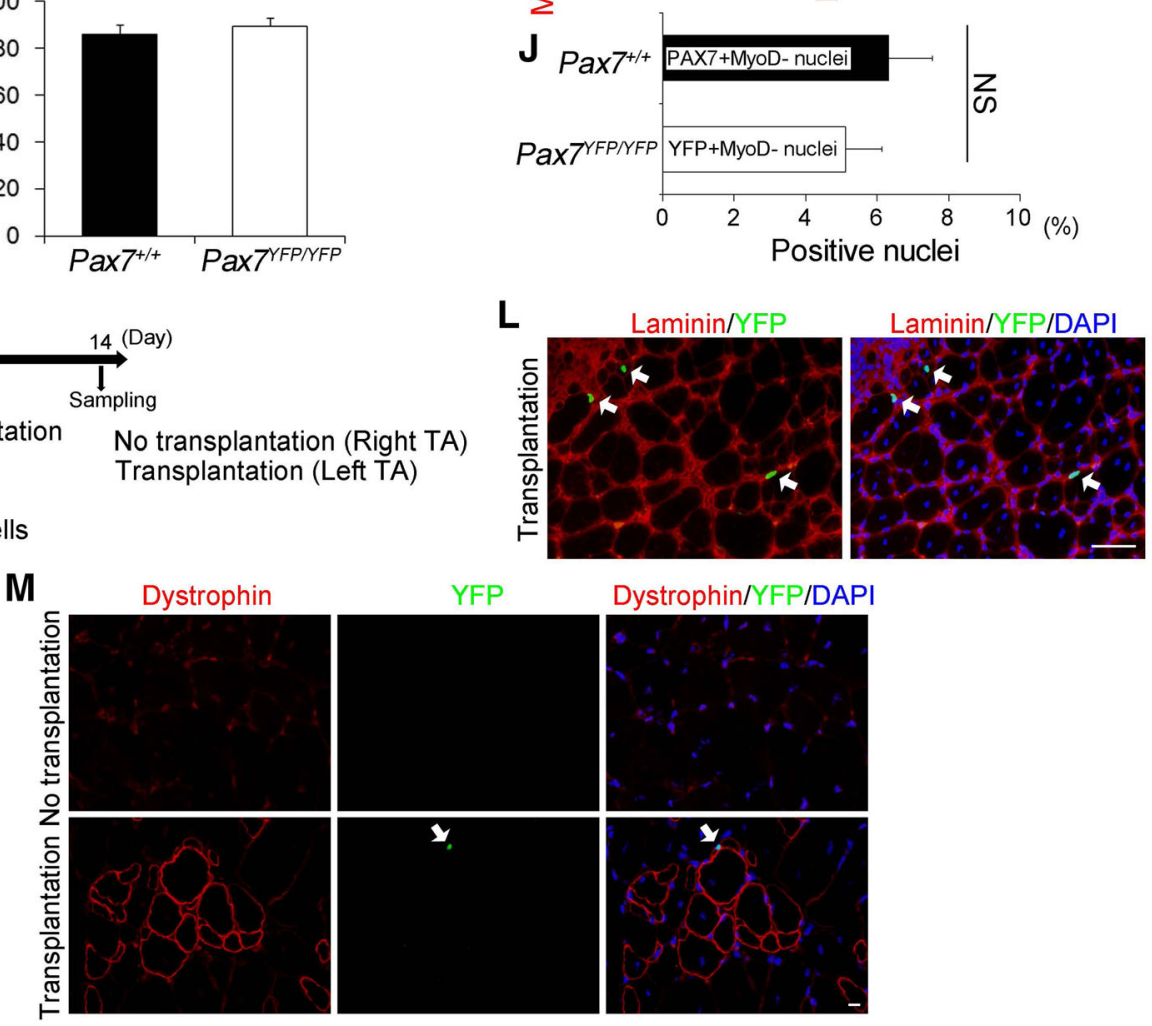

Fig. 4 (See legend on next page.) 
(See figure on previous page.)

Fig. 4 Homozygous Pax7-YFP mouse-derived satellite cells exhibit a wild-type stem cell function. a-c Regeneration was induced in the TA muscle of $\mathrm{Pax} 7^{+/+}$, Pax $7^{\mathrm{YFP} /+}$, and Pax ${ }^{\text {YFP } / F P}$ mice by injection of CTX as shown in Fig. 3. Regenerating muscles were isolated at day 14 following CTX injection. Representative immunohistochemical images for Laminin a2 in cross-sections of TA muscle (a). Cross-sectional area (CSA) of centrally nucleated regenerating myofibers was quantified $(\mathbf{b})$, scale bar $50 \mu \mathrm{m}$. $(n=4)$. Muscle weight of TA muscles was measured $(\mathbf{c})(n=3-7)$. Data represent means \pm s.e.m. One-way ANOVA followed by the Bonferroni post hoc test: NS non-significant. $\mathbf{d}$-j Satellite cell populations were isolated from limb muscles of Pax $7^{+/+}$or Pax $7^{\text {YFP MFP }}$ mice by FACS and cultured as shown in Fig. 2. Time course for analysis (d). e Representative images of EdU staining of plated satellite cells in GM conditions [quantified in (f)]. Nuclei were stained with DAPI. > 300 cells per mouse were counted ( $n=3$ mice). Data represent means \pm s.e.m. (NS non-significant), scale bar $50 \mu \mathrm{m}$. $\mathbf{g}$ Myogenic differentiation was induced under differentiation medium (DM) conditions for 4 days and cells immunostained for MyHC [differentiation index (h) was measured as the ratio of MyHC+ nuclei per total DAPI+ nuclei. $>200$ cells per mouse were counted ( $n=6-7$ mice)], scale bar $20 \mu \mathrm{m}$. Data represent means \pm s.d. (NS nonsignificant). i, $\mathbf{j}$ Self-renewal of satellite cells was induced under DM conditions for 4 days. (i) Co-immunostaining for PAX7 (or YFP) and MyoD. Arrows indicate Pax $7^{+}$(or YFP+)/MyoD ${ }^{-}$self-renewal cells [quantified in (j)], scale bar $10 \mu \mathrm{m}$. $>250$ nuclei per mouse $(n=3$ mice) were counted. Data represent means \pm s.d. (NS non-significant). $\mathbf{k}-\mathbf{m}$ To evaluate whether satellite cells isolated from Pax $7^{\text {YFP/YFP }}$ mice were able to give rise to progeny and regenerate muscle, transplantation analysis was performed. $\mathbf{k}$ Time course for analysis of transplantation. YFP ${ }^{+}$cells $\left(5 \times 10^{4}\right.$ cells) were transplanted into CTX pre-injured TA muscle of $\mathrm{mdx}$ mice $(n=4)$. The contralateral legs were injected with PBS and used as a control. Muscles were removed for immunohistochemistry 14 days post-transplantation. I Immunohistochemistry for YFP and laminin a 2 in cross-sections of TA muscles engrafted with YFP+ satellite cells. Arrows indicate YFP ${ }^{+}$cells, scale bar $50 \mu \mathrm{m}$. $\mathbf{m}$ Immunohistochemistry of dystrophin and YFP proteins in TA muscle of mdx mice $(n=4)$. Pax7-YFP homozygous mouse-derived satellite cells were capable of producing self-renewed cells and restoring dystrophin in mdx mice. Arrows indicate $\mathrm{YFP}^{+}$cells, scale bar $10 \mu \mathrm{m}$

applicable for in vitro and in vivo live cell-imaging analysis of satellite cell dynamics via YFP fluorescence. Because Pax7-YFP mouse-derived satellite cells express YFP-tagged PAX7 protein, they can also be used for ChIP-seq analysis to identify PAX7-regulated genes. Recent studies have described the functions of Pax7 in muscle diseases: PAX7-target genes are globally repressed by the DUX4 transcription factor, which is ectopically expressed in muscles of facioscapulohumeral muscular dystrophy (FSHD) patients [45]. Furthermore, in a mouse model of cancer cachexia, PAX7 protein is highly upregulated, which suppresses myogenic differentiation of satellite cells, leading to muscle atrophy [46]. Therefore, we hope that our Pax7-YFP mouse line will facilitate investigation of satellite cell biology and will benefit the development of stem-cell-based therapies to treat muscle diseases.

\section{Additional file}

Additional file 1: Table S1. Primers for quantitative PCR. (PDF 22 kb)

\begin{abstract}
Abbreviations
CSA: Cross-sectional area; CTX: Cardiotoxin; DM: Differentiation medium; DMD: Duchenne muscular dystrophy; EDL: Extensor digitorum longus; FACS: Fluorescence-activated cell sorting; FSHD: Facioscapulohumeral muscular dystrophy; GM: Growth medium; PFA: Paraformaldehyde; QPCR: Quantitative revere transcription-PCR; Sol: Soleus; TA: Tibialis anterior; YFP: Yellow fluorescent protein
\end{abstract}

\section{Acknowledgements}

We thank all lab members for technical support.

\section{Funding}

This work was supported by the Japan Science and Technology Agency (JST), the Japan Agency for Medical Research and Development (AMED, 16bm0704010h0001; 18ek0109383h0001), and the Grant-in-Aid for Scientific Research KAKENHI (15H05368, 15K16486, 16K13046, 18H03193,

18K17857, and 18K19749). This work was also supported, in part, by a Grant-
in-Aid for JSPS Research Fellows (16J00431) and the Takeda Science Foundation.

\section{Availability of data and materials}

All data generated and analyzed during the study are available from the corresponding author on a reasonable request.

\section{Authors' contributions}

YK performed the experiments and analyzed the data and wrote the manuscript. YO designed the experiments and interpreted the data, assembled the input data, and wrote the manuscript. All authors discussed the results and implications and commented on the manuscript. Both authors read and approved the final manuscript.

\section{Ethics approval}

All animal experimentation used in this study were approved by the Experimental Animal Care and Use Committee of Nagasaki University (ref. no. 1203190970).

\section{Consent for publication}

All authors have read the final version of the manuscript and consented to its submission to Skeletal Muscle.

\section{Competing interests}

The authors declare that they have no competing interests.

\section{Publisher's Note}

Springer Nature remains neutral with regard to jurisdictional claims in published maps and institutional affiliations.

\section{Author details \\ ${ }^{1}$ Musculoskeletal Molecular Biology Research Group, Basic and Translational Research Center for Hard Tissue Disease, Nagasaki University Graduate School of Biomedical Sciences, 1-7-1 Sakamoto, Nagasaki 852-8588, Japan. 2Japan Society for the Promotion of Science, 5-3-1 Kojimachi, Chiyoda-ku, Tokyo 102-0083, Japan. ${ }^{3}$ Agency for Medical Research and Development (AMED), 1-7-1 Otemachi, Chiyodaku, Tokyo 100-0004, Japan.}

Received: 9 May 2018 Accepted: 1 August 2018

Published online: 24 August 2018

\section{References}

1. Yin H, Price F, Rudnicki MA. Satellite cells and the muscle stem cell niche. Physiol Rev. 2013;93:23-67. 
2. Relaix F, Zammit PS. Satellite cells are essential for skeletal muscle regeneration: the cell on the edge returns centre stage. Development. 2012; 139:2845-56.

3. Brack AS, Rando TA. Tissue-specific stem cells: lessons from the skeletal muscle satellite cell. Cell Stem Cell. 2012;10:504-14.

4. Tierney MT, Sacco A. Satellite cell heterogeneity in skeletal muscle homeostasis. Trends Cell Biol. 2016;26:434-44.

5. Ono Y. Satellite cell heterogeneity and hierarchy in skeletal muscle. J Phys Fitness Sports Med. 2014;3:229-34.

6. Blau HM, Cosgrove BD, Ho AT. The central role of muscle stem cells in regenerative failure with aging. Nat Med. 2015;21:854-62.

7. Mauro A. Satellite cell of skeletal muscle fibers. J Biophys Biochem Cytol. 1961;9:493-5

8. Collins CA, Olsen I, Zammit PS, Heslop L, Petrie A, Partridge TA, Morgan JE. Stem cell function, self-renewal, and behavioral heterogeneity of cells from the adult muscle satellite cell niche. Cell. 2005;122:289-301.

9. Boldrin L, Zammit PS, Muntoni F, Morgan JE. Mature adult dystrophic mouse muscle environment does not impede efficient engrafted satellite cell regeneration and self-renewal. Stem Cells. 2009;27:2478-87.

10. Ono Y, Boldrin L, Knopp P, Morgan JE, Zammit PS. Muscle satellite cells are a functionally heterogeneous population in both somite-derived and branchiomeric muscles. Dev Biol. 2010;337:29-41.

11. Sherwood Rl, Christensen JL, Conboy IM, Conboy MJ, Rando TA, Weissman IL, Wagers AJ. Isolation of adult mouse myogenic progenitors: functional heterogeneity of cells within and engrafting skeletal muscle. Cell. 2004;119: 543-54

12. Sacco A, Doyonnas R, Kraft P, Vitorovic S, Blau HM. Self-renewal and expansion of single transplanted muscle stem cells. Nature. 2008;456:502-6.

13. Ono Y, Masuda S, Nam HS, Benezra R, Miyagoe-Suzuki Y, Takeda S. Slowdividing satellite cells retain long-term self-renewal ability in adult muscle. J Cell Sci. 2012;125:1309-17.

14. McCarthy JJ, Mula J, Miyazaki M, Erfani R, Garrison K, Farooqui AB, Srikuea R, Lawson BA, Grimes B, Keller C, et al. Effective fiber hypertrophy in satellite cell-depleted skeletal muscle. Development. 2011;138:3657-66.

15. Murphy MM, Lawson JA, Mathew SJ, Hutcheson DA, Kardon G. Satellite cells, connective tissue fibroblasts and their interactions are crucial for muscle regeneration. Development. 2011;138:3625-37.

16. Lepper C, Partridge TA, Fan CM. An absolute requirement for Pax7-positive satellite cells in acute injury-induced skeletal muscle regeneration. Development. 2011;138:3639-46.

17. Sacco A, Mourkioti F, Tran R, Choi J, Llewellyn M, Kraft P, Shkreli M, Delp S, Pomerantz JH, Artandi SE, Blau HM. Short telomeres and stem cell exhaustion model Duchenne muscular dystrophy in $\mathrm{mdx} / \mathrm{mTR}$ mice. Cell. 2010;143:1059-71.

18. Halevy O, Piestun $Y$, Allouh MZ, Rosser BW, Rinkevich $Y$, Reshef $R$, Rozenboim I, Wleklinski-Lee M, Yablonka-Reuveni Z. Pattern of Pax7 expression during myogenesis in the posthatch chicken establishes a model for satellite cell differentiation and renewal. Dev Dyn. 2004:231:489-502.

19. Olguin HC, Olwin BB. Pax-7 up-regulation inhibits myogenesis and cell cycle progression in satellite cells: a potential mechanism for self-renewal. Dev Biol. 2004;275:375-88.

20. Zammit PS, Golding JP, Nagata Y, Hudon V, Partridge TA, Beauchamp JR. Muscle satellite cells adopt divergent fates: a mechanism for self-renewal? J Cell Biol. 2004;166:347-57.

21. Montarras D, Morgan J, Collins C, Relaix F, Zaffran S, Cumano A, Partridge T, Buckingham M. Direct isolation of satellite cells for skeletal muscle regeneration. Science. 2005;309:2064-7.

22. Relaix F, Rocancourt D, Mansouri A, Buckingham M. Divergent functions of murine Pax3 and Pax7 in limb muscle development. Genes Dev. 2004;18:1088-105.

23. Oustanina S, Hause G, Braun T. Pax7 directs postnatal renewal and propagation of myogenic satellite cells but not their specification. EMBO J. 2004;23:3430-9

24. Relaix F, Rocancourt D, Mansouri A, Buckingham M. A Pax3/Pax7-dependent population of skeletal muscle progenitor cells. Nature. 2005;435:948-53.

25. Kuang S, Charge SB, Seale P, Huh M, Rudnicki MA. Distinct roles for Pax7 and Pax3 in adult regenerative myogenesis. J Cell Biol. 2006;172:103-13.

26. Zammit PS, Relaix F, Nagata Y, Ruiz AP, Collins CA, Partridge TA, Beauchamp JR. Pax7 and myogenic progression in skeletal muscle satellite cells. J Cell Sci. 2006:119:1824-32.
27. Seale P, Sabourin LA, Girgis-Gabardo A, Mansouri A, Gruss P, Rudnicki MA. Pax7 is required for the specification of myogenic satellite cells. Cell. 2000; 102:777-86

28. Magli A, Incitti T, Kiley J, Swanson SA, Darabi R, Rinaldi F, Selvaraj S, Yamamoto A, Tolar J, Yuan C, et al. PAX7 targets, CD54, integrin alpha9beta1, and SDC2, allow isolation of human ESC/iPSC-derived myogenic progenitors. Cell Rep. 2017;19:2867-77.

29. Relaix F, Montarras D, Zaffran S, Gayraud-Morel B, Rocancourt D, Tajbakhsh S, Mansouri A, Cumano A, Buckingham M. Pax3 and Pax7 have distinct and overlapping functions in adult muscle progenitor cells. J Cell Biol. 2006;172: 91-102.

30. von Maltzahn J, Jones AE, Parks RJ, Rudnicki MA. Pax7 is critical for the normal function of satellite cells in adult skeletal muscle. Proc Natl Acad Sci U S A. 2013;110:16474-9.

31. Gunther S, Kim J, Kostin S, Lepper C, Fan CM, Braun T. Myf5-positive satellite cells contribute to Pax7-dependent long-term maintenance of adult muscle stem cells. Cell Stem Cell. 2013;13:590-601.

32. Pasut A, Chang NC, Gurriaran-Rodriguez U, Faulkes S, Yin H, Lacaria M, Ming $\mathrm{H}$, Rudnicki MA. Notch signaling rescues loss of satellite cells lacking Pax7 and promotes brown adipogenic differentiation. Cell Rep. 2016;16:333-43.

33. Lepper C, Conway SJ, Fan CM. Adult satellite cells and embryonic muscle progenitors have distinct genetic requirements. Nature. 2009;460:627-31.

34. Bosnakovski D, Xu Z, Li W, Thet S, Cleaver O, Perlingeiro RC, Kyba M. Prospective isolation of skeletal muscle stem cells with a Pax7 reporter. Stem Cells. 2008;26:3194-204.

35. Rocheteau P, Gayraud-Morel B, Siegl-Cachedenier I, Blasco MA, Tajbakhsh S. A subpopulation of adult skeletal muscle stem cells retains all template DNA strands after cell division. Cell. 2012;148:112-25.

36. Sampath SC, Sampath SC, Ho ATV, Corbel SY, Millstone JD, Lamb J, Walker J, Kinzel B, Schmedt C, Blau HM. Induction of muscle stem cell quiescence by the secreted niche factor Oncostatin M. Nat Commun. 2018;9:1531.

37. Kitajima Y, Ogawa S, Ono Y. Visualizing the functional heterogeneity of muscle stem cells. Methods Mol Biol. 2016:1516:183-93.

38. Ono Y, Calhabeu F, Morgan JE, Katagiri T, Amthor H, Zammit PS. BMP signalling permits population expansion by preventing premature myogenic differentiation in muscle satellite cells. Cell Death Differ. 2011;18:222-34.

39. Lepper C, Fan CM. Inducible lineage tracing of Pax7-descendant cells reveals embryonic origin of adult satellite cells. Genesis. 2010;48:424-36.

40. Murdoch B, DelConte C, Garcia-Castro MI. Pax7 lineage contributions to the mammalian neural crest. PLoS One. 2012;7:e41089.

41. Liu L, Cheung TH, Charville GW, Rando TA. Isolation of skeletal muscle stem cells by fluorescence-activated cell sorting. Nat Protoc. 2015;10:1612-24.

42. Maesner CC, Almada AE, Wagers AJ. Established cell surface markers efficiently isolate highly overlapping populations of skeletal muscle satellite cells by fluorescence-activated cell sorting. Skelet Muscle. 2016;6:35.

43. Fukada S, Uezumi A, Ikemoto M, Masuda S, Segawa M, Tanimura N, Yamamoto H, Miyagoe-Suzuki Y, Takeda S. Molecular signature of quiescent satellite cells in adult skeletal muscle. Stem Cells. 2007;25:2448-59.

44. Ono Y, Urata Y, Goto S, Nakagawa S, Humbert PO, Li TS, Zammit PS. Muscle stem cell fate is controlled by the cell-polarity protein Scrib. Cell Rep. 2015; 10:1135-48.

45. Banerji CRS, Panamarova M, Hebaishi $H$, White RB, Relaix F, Severini $S$, Zammit PS. PAX7 target genes are globally repressed in facioscapulohumeral muscular dystrophy skeletal muscle. Nat Commun. 2017:8:2152.

46. He WA, Berardi E, Cardillo VM, Acharyya S, Aulino P, Thomas-Ahner J, Wang J. Bloomston M, Muscarella P, Nau P, et al. NF-kappaB-mediated Pax7 dysregulation in the muscle microenvironment promotes cancer cachexia. J Clin Invest. 2013;123:4821-35 